\title{
Vasculopatia polipoidal idiopática da coróide
}

\author{
Idiopathic polypoidal choroidal vasculopathy
}

\author{
Rafael Ernane Andrade ${ }^{1}$ \\ André Castelo Branco ${ }^{2}$ \\ Rogério Alves Costa ${ }^{2}$ \\ Fausto Uno ${ }^{3}$ \\ Maurício Nakanami $^{4}$ \\ Tércio Guia ${ }^{5}$ \\ Michel Eid Farah ${ }^{6}$
}

\section{RESUMO}

Objetivo: Descrever os achados da tomografia de coerência óptica, angiofluoresceinografia e indocianinografia na vasculopatia polipoidal idiopática da coróide. Métodos: Realizou-se análise criteriosa dos exames complementares de angiofluoresceinografia e indocianinografia, comumente utilizados para o diagnóstico, assim como da tomografia de coerência óptica, em quatro olhos de uma série de três pacientes com vasculopatia polipoidal idiopática da coróide. Resultados: Os quatro olhos mostraram lesões subretinianas vermelho-alaranjadas, sendo que dois casos apresentaram descolamento hemorrágico do epitélio pigmentado da retina, além de manifestações exsudativas e hemorrágicas associadas. A angiofluoresceinografia revelou dilatações aneurismáticas em ramificações anormais de vasos da coróide em apenas 1 caso, ao contrário da indocianinografia que claramente demonstrou as lesões em todos os casos. A tomografia de coerência óptica confirmou descolamento hemorrágico espontâneo do epitélio pigmentado da retina em 2 casos. Conclusão: A indocianinografia é o exame de escolha no diagnóstico da vasculopatia polipoidal da coróide, mas em alguns casos a associação com a tomografia de coerência óptica e angiofluoresceinografia pode ser útil no diagnóstico e seguimento.

Descritores: Coróide/irrigação sangüínea; Hemorragia da coróide/etiologia; Doenças da coróide/patologia; Angiofluoresceinografia; Verde indocianina/uso diagnóstico; Fundo de olho; Tomografia computadorizada por raios- $x$

Trabalho realizado no setor de Retina e Vítreo do Departamento de Oftalmologia, Universidade Federal de São Paulo/Escola Paulista de Medicina (UNIFESP/EPM).

${ }^{1}$ Estagiário do $2^{\circ}$ ano do setor de Retina e Vítreo Universidade Federal de São Paulo/Escola Paulista de Medicina (UNIFESP/EPM).

${ }^{2}$ Pós-graduando nível doutorado do setor de Retina e Vítreo Universidade Federal de São Paulo/Escola Paulista de Medicina (UNIFESP/EPM).

${ }^{3}$ Mestre em Oftalmologia Universidade Federal de São Paulo/Escola Paulista de Medicina (UNIFESP/EPM).

${ }^{4}$ Médico colaborador do Setor de Retina e Vítreo Universidade Federal de São Paulo. Escola Paulista de Medicina (UNIFESP/EPM).

${ }^{5}$ Tecnólogo do setor de Retina e Vítreo Universidade Federal de São Paulo/Escola Paulista de Medicina (UNIFESP/EPM).

${ }^{6}$ Professor Livre-Docente em Oftalmologia Universidade Federal de São Paulo/Escola Paulista de Medicina (UNIFESP/EPM).

Endereço para correspondência: Av. Dr. Altino Arantes, 574, apto. 83 - São Paulo (SP) CEP 04042-003. E-mail: rafaelernane@uol.com.br

Recebido para publicação em 21.09.2001

Aceito para publicação em 16.01.2002

Nota Editorial: Pela análise deste trabalho e por sua anuência na divulgação desta nota, agradecemos aos Drs. Eduardo Cunha de Souza e João Luis Lobo Ferreira. $\frac{\text { INTRODUÇÃOO }}{\text { A vasculopatia polipoidal idiopática da coróide }{ }^{(1)} \text {, anteriormente conhe- }}$

A vasculopatia polipoidal idiopática da coróide ${ }^{(1)}$, anteriormente conhecida como síndrome da hemorragia uveal posterior ${ }^{(2)}$ ou síndrome dos múltiplos descolamentos recorrentes serosanguinolentos do epitélio pigmentado da retina ${ }^{(3-4)}$, é uma entidade clínica que vem recentemente merecendo enfoque especial no campo das maculopatias exsudativas. Consiste em uma ramificação anormal com dilatações marginais dos vasos da coróide interna que se manifesta, geralmente, como lesões subretinianas elevadas, vermelho-alaranjadas, associadas a um descolamento serosangüíneo recorrente do epitélio pigmentado da retina ${ }^{(5)}$.

Primariamente considerou-se que estas alterações vasculares eram peripapilares, embora Yannuzzi e col. tenham demonstrado alterações maculares $^{(6)}$, e mais raramente, periféricas ${ }^{(7)}$. Epidemiologicamente achava-se que a doença era restrita à população negra, mas novos estudos mostraram que esta alteração podia atingir qualquer raça, tendo maior predileção pelas populações mais pigmentadas (negros, pardos e asiáticos) ${ }^{(8)}$.

A indocianinografia é o exame de escolha para o diagnóstico por meio da identificação dos componentes vasculares anormais da vasculopatia polipoidal idiopática da coróide ${ }^{(5)}$.

Em muitos casos o quadro clínico tende a ter resolução espontânea e o 
prognóstico visual geralmente é bom, porém, em casos selecionados, a fotocoagulação a laser da estrutura polipoidal pode resultar em resolução das complicações serosanguinolentas ${ }^{(9-10)}$.

Iijima e col. demonstraram por meio da tomografia de coerência óptica, exame que realiza cortes seccionais da retina e coróide, as complicações associadas à patologia em questão, porém careceu em demonstrar achados típicos da vasculopatia polipoidal idiopática da coróide em relatos na literatura ${ }^{(11)}$.

Yannuzzi e col. observaram que 7,8\% dos pacientes com maculopatia exsudativa e diagnóstico prévio de degeneração macular relacionada à idade tinham na verdade vasculopatia polipoidal idiopática da coróide ${ }^{(12)}$. Mais recentemente, os mesmos autores evidenciaram vasculopatia polipoidal idiopática da coróide em alguns pacientes com o diagnóstico prévio de coroidopatia serosa central ${ }^{(13)}$.

Este trabalho objetiva descrever os achados angiofluoresceinográficos, indocianinográficos e da tomografia de coerência óptica em três pacientes com vasculopatia polipoidal idiopática da coróide.

\section{MÉTODOS}

Foram estudados prospectivamente quatro olhos de três pacientes com maculopatia exsudativa e suspeita diagnóstica de vasculopatia polipoidal idiopática da coróide, no setor de Retina e Vítreo da Escola Paulista de Medicina-UNIFESP, onde foram realizadas: oftalmoscopia binocular indireta com lente asférica de 20 dioptrias, biomicroscopia de fundo com lente de contato de Goldmann, angiofluoresceinografia, indocianinografia e tomografia de coerência óptica.

\section{RELATO DE CASOS}

\section{Caso 1}

Paciente de 67 anos, sexo feminino, preta. Queixa de embaçamento visual no olho esquerdo (OE) há 25 dias, com piora progressiva. Refere um episódio prévio de baixa da visão neste olho há 6 anos. Tem diagnóstico de diabetes mellitus insulino-dependente há 9 anos e hipertensão arterial sistêmica há 12 anos, controlada com metil-dopa e hidroclorotiazida. A acuidade visual com a melhor correção foi no olho direito (OD) de 20/60 e no OE de 20/400. A tela de Amsler mostrava metamorfopsia e escotoma central relativo no OE. A biomicroscopia anterior revelou catarata incipiente em ambos os olhos (AO). A fundoscopia apresentou no OD a presença de membrana epirretiniana macular, e no OE na região peripapilar uma lesão vermelho-alaranjada e elevada, associada à hemorragia sub-retiniana e despigmentação do epitélio pigmentado da retina, também presente no pólo posterior. A angiofluoresceinografia revela ramificação e dilatação de vasos coroidais na região peripapilar e hipofluorescência por bloqueio devido à hemorragia sub-retiniana na borda nasal da lesão, e hiperfluo- rescência por defeito em janela perimacular. A indocianinografia mostrou essas dilatações e ramificações dos vasos peripapilares da coróide de forma mais evidente. A tomografia de coerência óptica revelou área de descolamento do epitélio pigmentado da retina sensorial associado a uma descontinuidade na camada de alta refletividade correspondente ao complexo epitélio pigmentado da retina e coriocapilar, consistindo na superfície anterior da lesão polipoidal (Figura 1 A, B, C, D).

\section{Caso 2}

75 anos, sexo feminino, branca. Queixa de embaçamento visual em OE há 2 anos, com piora há 1 mês. História de facectomia com implante de lente intra-ocular em OD há 4 anos e em OE há 3 anos. A acuidade visual com a melhor correção foi de 20/20 no OD e 20/200 no OE. A biomicroscopia anterior revelou pseudofacia em ambos os olhos. O teste com tela de Amsler demonstrou escotoma central relativo no OE. A oftalmoscopia revelou lesão vermelho-alaranjada peripapilar associada a descolamento hemorrágico do epitélio pigmentado da retina e a áreas extensas de hemorragia sub-retiniana atingindo a região macular. A angiofluoresceinografia no $\mathrm{OE}$ apresentou região de hipofluorescência por bloqueio oriunda de hemorragia sub-retiniana, além de lesão hiperfluorescente peripapilar por vazamento. A indocianinografia demonstrou vasos dilatados multifocais peripapilares da coróide que vazavam nas fases tardias. A tomografia de coerência óptica mostrou áreas de descolamento hemorrágico do epitélio pigmentado da retina, contíguas com a imagem da borda anterior da massa polipoidal.

\section{Caso 3}

Paciente de 43 anos, sexo feminino, branca. Queixa de baixa súbita da visão há 3 anos. A acuidade visual com a melhor correção foi de 20/100 no OD e 20/20 no OE. O teste com tela de Amsler revelou metamorfopsia no OD, não detectando no OE. A oftalmoscopia apresentou no OD lesão elevada, alaranjada, sub-retiniana, nasal inferior à fóvea, e no $\mathrm{OE}$ mostrou uma lesão elevada, cinza-alaranjada, na região temporal inferior, próximo a arcada vascular temporal inferior. A angiofluoresceinografia revelou na mácula do $\mathrm{OD}$ e na região temporal inferior do $\mathrm{OE}$ lesões hiperfluorescentes com discreto vazamento. Ambas as lesões foram melhor observadas na indocianinografia como áreas de pontos quentes, consistindo em pequenos vasos dilatados da coróide, mais evidentes nas fases tardias. A tomografia de coerência óptica não mostrou áreas de descolamento do epitélio pigmentado da retina nos locais correspondentes às lesões descritas, conservando a depressão foveal normal em ambos os olhos.

\section{DISCUSSÃO}

A vasculopatia polipoidal idiopática da coróide é uma entidade clínica descrita por Yannuzzi em 1982 ${ }^{(1)}$. É caracterizada por uma rede vascular ramificada de vasos da coróide interna com dilatações marginais, formando estruturas de pólipos, que se 

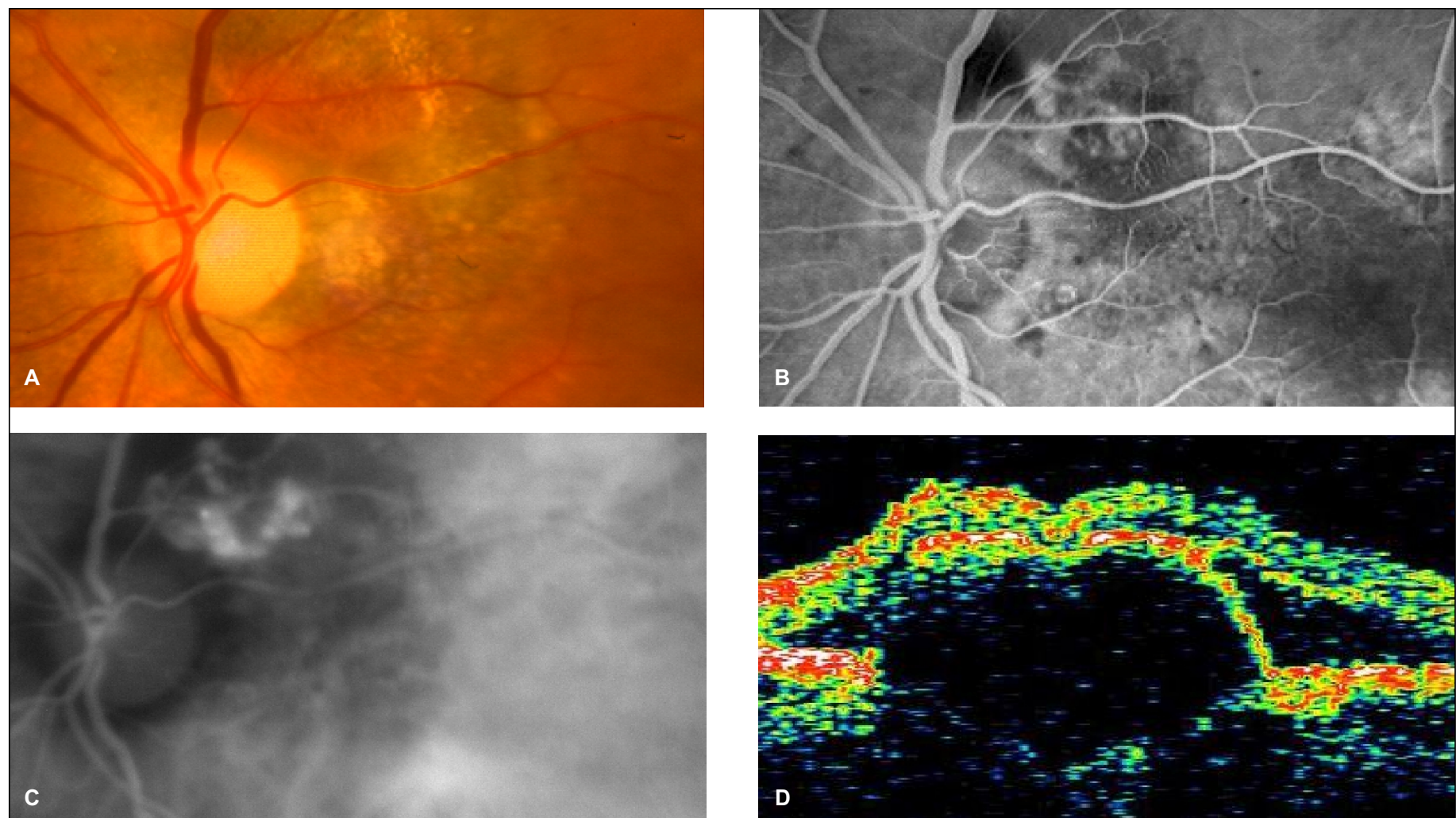

Figura 1 - Caso 1; A) Retinografia do OE mostra lesão elevada vermelho-alaranjada, sub-retiniana na região peripapilar superior e alteraç̃es do EPR na região macular superior e inferior; B) Angiofluoresceinografia revela vaso dilatado e ramificado da coróide na região peripapilar e hipofluorescência por bloqueio na borda nasal da lesão, e hiperfluorescência por defeito em janela perimacular; C) Indocianinografia detecta a disposição polipoidal da lesão peripapilar, com dilatação e ramificação do vaso coroidal. A linha branca pontilhada é a esquematização do corte da tomografia de coerência óptica; D) Corte horizontal de tomografia de coerência óptica sobre a lesão, permite a observação de área de descolamento protuso do epitélio pigmentado da retina associado a descolamento da retina sensorial.

traduzem na fundoscopia como lesões elevadas sub-retinianas, vermelho-alaranjadas, associadas com episódios recorrentes de descolamento sero-hemorrágico do epitélio pigmentado da reti$\mathrm{na}^{(5)}$. Anteriormente foi conhecida como "doença das mulheres pretas" ${ }^{(3)}$, por se acreditar que esta seria restrita à raça melanodérmica, todavia, recentes estudos demostraram que a raça branca e o sexo masculino são também acometidos, havendo, porém, uma certa predileção pelo sexo feminino e pelas raças pigmentadas (pretos, pardos e asiáticos) ${ }^{(8)}$. Todos os casos apresentados nesta série em estudo foram do sexo feminino, sendo dois da raça branca. Esta doença é mais freqüente na faixa etária entre 40 e 80 anos, sendo esses dados compatíveis com os casos aqui descritos (43 a 75 anos).

A sintomatologia variou desde um leve embaçamento até a baixa de visão acentuada (20/20 à 20/400), tendo ocorrido dois casos unilaterais e um bilateral. Apesar da maioria dos relatos já descritos terem mostrado bom prognóstico visual ${ }^{(5-6)}$, cursando, muitas vezes, com a involução espontânea das lesões, a acuidade visual pode estar bastante reduzida quando existe envolvimento macular, principalmente foveal por hemorragia ou exsudação, como no caso 2 aqui apresentado.

O diagnóstico da vasculopatia polipoidal idiopática da coróide pode ser confirmado pela angiofluoresceinografia, como no caso 1 , onde podem ser observadas as dilatações aneurismáticas dos vasos coroidais peripapilares, com vazamento nas fases mais tardias, principalmente se forem de grandes dimensões e associadas a atrofia do epitélio pigmentado da retina sobrejacente. $\mathrm{Na}$ indocianinografia as lesões foram melhor definidas, por se tratar de uma doença primária da coróide, e por estar freqüentemente associada a hemorragia e ao descolamento recorrente do epitélio pigmentado da retina, sendo considerada pela maioria dos autores como exame de escolha para essa entidade, já que pode demonstrar as múltiplas áreas de dilatações aneurismáticas e ramificadas dos vasos da coróide de médio e grande calibre como no caso 1 e 2, e pontos quentes como no caso 3 , servindo como guia, se indicado, para tratamento com fotocoagulação a laser ${ }^{(10)}$.

A tomografia de coerência óptica revelou áreas de descolamento hemorrágico do epitélio pigmentado e da retina sensorial, caracterizadas por uma proeminente protusão destas estruturas, associada a uma descontinuidade na camada de alta refletividade que delineia a borda anterior dos elementos vasculares polipoidais da coróide, podendo então, auxiliar na compreensão, diagnóstico e acompanhamento da evolução dessas alterações ainda pouco conhecidas.

Este estudo é a primeira descrição da vasculopatia polipoidal idiopática da coróide na literatura nacional, sendo um importante diagnóstico diferencial das maculopatias exsudati- 
vas, incluindo a degeneração macular relacionada à idade ${ }^{(12)} \mathrm{e}$ coroidopatia serosa central ${ }^{(13)}$, onde a diferenciação torna-se de suma importância devido a história natural e tratamentos específicos.

\section{ABSTRACT}

Purpose: To describe the role of the optical coherence tomography, fluorescein and indocyanine green angiography findings in the diagnosis of idiopathic polypoidal choroidal vasculopathy (IPCV). Methods: A series of four eyes of three patients thought to have IPCV were examined and evaluated by optical coherence tomography, fluorescein and indocyanine green angiographies. Results: Fluorescein angiography was highly important for the diagnosis only in case 1. Indocyanine green angiography showed a branching network of the vessels with marginal vascular dilatations in all cases. Optical coherence tomography showed hemorrhagic detachment of the retinal pigment epithelium contiguous with the anterior delimitation of the polypoidal elements in two cases. Conclusion: The association of clinical manifestations with fluorescein and indocyanine green angiographies and optical coherence tomography can be useful in the differential diagnosis of exudative maculopathies, particularly IPCV with serohemorrhagic manifestations.

Keywords: Choroid/blood supply; Choroid hemorrhage/ etiology; Choroid diseases/pathology; Fluorescein angiography; Indocyanine green/diagnostic use; Fundus oculi; X-ray computed tomography

\section{REFERÊNCIAS}

1. Yannuzzi LA. Idiopathic polypoidal choroidal vasculopathy. In: Macula Society Meeting 1982. Miami, Fla. [cited on 2002 Abr 30]. Available from URL: http://www.wilmer.jhu.edu/training/profrounds/CASE12/case12.html

2. Kleiner RC, Brucker AJ, Johnston RL. The posterior uveal bleeding syndrome. Retina 1990;10:9-17.

3. Stern RM, Zakov ZN, Zegarra H, Gutman FA. Multiple recurrent serosanguineous retinal pigment epithelial detachments in black women. Am J Ophthalmol 1985;100:560-9.

4. Perkovich BT, Zakov ZN, Berlin LA, Weidenthal D, Avins LR. An update on multiple recurrent serosanguineous retinal pigment epithelial detachments in black women. Retina 1990;10:18-26.

5. Yannuzzi LA, Sorenson J, Spaide RF, Lipson B. Idiopathic polypoidal choroidal vasculopathy (IPCV). Retina 1990;10:1-8.

6. Yannuzzi LA, Ciardella A, Spaide RF, Rabb M, Freund KB, Orlock DA. The expanding clinical spectrum of idiopathic polypoidal choroidal vasculopathy. Arch Ophthalmol 1997;115:478-85.

7. Yannuzzi LA, Nogueira F, Spaide R, Guyer DR, Orlock DA, Colombero D, et al. Idiopathic polypoidal choroidal vasculopathy: a peripheral lesion. Arch Ophthalmol 1998;116:382-3.

8. Moorthy RS, Lyon AT, Rabb MF, Spaide RF, Yannuzzi LA, Jampol LM. Idiopathic polypoidal choroidal vasculopathy of the macula. Ophthalmology 1998;105:1380-5.

9. Spaide RF, Yannuzzi LA, Slakter JS, Sorenson J, Orlock DA. Indocyanine green videoangiography of polypoidal vasculopathy. Retina 1995;15:100-10.

10. MacCumber MW, Dastgheib K, Bressler NM, Chan C, Harris M, Fine S, et al. Clinicopathologic correlation of the multiple recurrent serosanguineous retinal pigment epithelial detachments syndrome. Retina 1994;14:143-52.

11. Iijima H, Imai M, Gohdo T, Tsukahara S. Optical coherence tomography of idiopathic polypoidal choroidal vasculopathy. Am J Ophthalmol 1999;127:301-5.

12. Yannuzzi LA, Wong DW, Sforzolini BS, Goldbaum M, Tang KC, Spaide RF, et al. Polypoidal choroidal vasculopathy and neovascularized age-related macular degeneration. Arch Ophthalmol 1999;117:1503-10.

13. Yannuzzi LA, Freund KB, Goldbaum M, Scassellati-Sforzolini B, Guyer DR, Spaide RF, et al. Polypoidal choroidal vasculopathy masquerading as central serous chorioretinopathy. Ophthalmology 2000;107:767-7.

\section{SIMPÓSIO INTERNACIONAL DA SANTA CASA DE PORTO ALEGRE}

12 e 13 dedulhode 2002

Porto Alogre-RS

INFORMAÇÕES: tel.: (51) 3214-8506 